\title{
Asparagus racemosus Root Extract and Isoprinosine Exhibits Radio-mitigating Activity against lonizing Radiation-induced Detrimental Effects in Swiss Albino Mice
}

\author{
Sharmila Kameyanda Poonacha', Satheesh Kumar Bhandary Bavabeedu², Suchetha Kumari Nalilu³, Pooja S4, Vadisha Srinivas Bhat ${ }^{2}$, \\ Ganesh Sanjeev ${ }^{5}$, Ronald Fernandes ${ }^{6, *}$ \\ 'Central Research Laboratory, KS Hegde Medical Academy, NITTE (Deemed to be University), Mangalore, Karnataka, INDIA. \\ 2Department of ENT, KS Hegde Medical Academy, NITTE (Deemed to be University), Mangalore, Karnataka, INDIA. \\ ${ }^{3}$ Department of Biochemistry, KS Hegde Medical Academy, NITTE (Deemed to be University), Mangalore, Karnataka, INDIA. \\ ${ }^{4}$ Central Research Laboratory, RAK Medical and Health Sciences University, Ras AI Khaimah, UAE. \\ 5Department of Physics, Mangalore University, Konaje, Mangalore, Karnataka, INDIA. \\ ${ }^{6}$ Department of Pharmaceutical Chemistry, NGSM Institute of Pharmaceutical Sciences, NITTE (Deemed to be University), Mangalore, Karnataka, INDIA.
}

\begin{abstract}
Objectives: To evaluate the radio - mitigating property of Asparagus racemosus root ethanolic extract and Isoprinosine against ionizing radiationinduced harmful effects in Swiss albino mice. Methods: The experimental animals were exposed to sublethal dose (6 Gy) of whole-body electron beam radiation and administered orally with Asparagus racemosus root ethanolic extract (ARE - 200mg/kg bd. wt) and Isoprinosine (IPR - 400 $\mathrm{mg} / \mathrm{kg}$ bd. wt) for 15 consecutive days. After the experimental period, the animals were sacrificed. Blood and liver tissue were collected. Blood sample was used for assessment of hematological parameters, serum was used for biochemical analysis (lipid peroxidation, total antioxidant capacity) and the level of pro-inflammatory cytokines was determined in the liver homogenate. Results: The radiation control group showed a statistically significant reduction in the hematological parameters. IPR post-treatment group showed a substantial increase in the percentage of lymphocytes and monocytes. ARE and IPR post-treatment caused a statistically significant improvement in platelet count. Irradiation of mice resulted in a significant elevation in lipid peroxidation. Post-treatment of mice with ARE and IPR caused significant depletion in lipid peroxidation. The serum total antioxidant capacity (TAC) in the irradiated group decreased significantly. Administration of ARE to the animals after irradiation caused a significant elevation in TAC level. IPR post-treatment did not show any substantial change in the reduced level of TAC. The cytokines level in the
\end{abstract}

liver homogenate of irradiated mice showed that Interleukin -2 (IL-2), Tumor Necrosis Factor alpha (TNF- $\alpha$ ) and Interferon gamma (IFN- - ) significantly increased after radiation exposure. Administration of ARE and IPR after irradiation significantly reduced the levels of IL-2 and TNF- $\alpha$ in the posttreatment groups. An elevated level of IFN- $\gamma$ was reduced considerably in the IPR post-treatment animals. Conclusion: Asparagus racemosus root ethanolic extract and Isoprinosine exhibit radio-mitigating effects against electron beam radiation - induced detrimental effects. This occurs through several mechanisms, such as free radical scavenging, anti-inflammation, facilitation of repair activity, regeneration of hematopoietic cells and affecting to molecular levels.

Key words: Asparagus racemosus, Isoprinosine, Radio-mitigation, lonizing radiation.

\section{Correspondence}

Dr. Ronald Fernandes

Professor, Department of Pharmaceutical Chemistry, NGSM Institute of Pharmaceutical Sciences, NITTE (Deemed to be University). Paneer, Deralakatte-575018, Mangalore, Karnataka, INDIA.

Phone: +919480115227

Email: ronaldfernandesv@gmail.com

DOI: 10.5530/jyp.2020.12.65

\section{INTRODUCTION}

It is well established that the ionizing radiations interact with biological tissues mainly by indirect effect and induce cellular damage primarily by the generation of free radicals or Reactive Oxygen Species (ROS) in the cellular environment. ${ }^{1}$ The ROS thus generated are highly reactive and interact with molecular oxygen or various vital biomolecules such as DNA, lipids, proteins and also with membranous structures of cells leading to oxidative stress. This process subsequently disrupts the homeostasis between the pro-oxidants and antioxidants inside the microenvironment of the cell that causes damage to cell components. ${ }^{2}$

Radiotherapy has emerged an important treatment modality for cancer, which depends on the stage of the tumor, its localization and the general health of the patient; it may be used as a single modality or as an adjuvant along with surgery and chemotherapy. ${ }^{3}$ However, effective use of ionizing radiation has become limited due to the side effects that result from radiation-induced damage to healthy tissue.
Radiation may cause bone marrow suppression and depletion of peripheral blood lymphocytes that leads to severely inhibit the function of the immune system, such as making the exposed animals susceptible to opportunistic pathogens, more easily to be infected and some time to be lethal. Prevalence of long-term immunosuppression also concerns in patients treated with radio or chemotherapy years after treatment. ${ }^{4-6}$

Radioprotectors are the compounds that are developed as a result of the undesirable consequences of radiation with the intent to reduce the damage in healthy tissues caused by radiation. These compounds which are rich in antioxidants must be present before or during radiation exposure for effectiveness. Other agents, termed mitigators, may be used to minimize toxicity after radiation exposure. ${ }^{7}$ Effective radioprotectors and radio-recovery drugs have to be developed which is of great importance in view of their potential application during both planned radiation exposure (radiotherapy) and unplanned radiation exposure

This is an open access article distributed under the terms of the Creative Commons Attribution-NonCommercial-ShareAlike 4.0 License, which allows others to remix, tweak, and build upon the work non-commercially, as long as the author is credited and the new creations are licensed under the identical terms. 
(used in the nuclear industry, natural background radiation, or other sources). Radiation-induced late normal tissue toxicity is considerably being recognized as a phenomenon of ongoing changes in tissue after radiation but before the manifestation of toxicity. These events include constant mitotic cell death and perpetually active cytokine cascades that leads to vascular damage, tissue hypoxia and excessive extracellular matrix deposition. Radiation mitigators strive to interrupt these cascades or intervene to prevent the perpetuation of damage and thus reduce the expression of toxicity. However, to date, no ideal agents are available as most synthetic compounds are toxic at their optimal concentrations. ${ }^{8-10}$ From time immemorial, medicinal plants served as a good source of pharmacological compounds and plant-based medicinal practices are effectively used in indigenous systems of medicine. Asparagus racemosus (Shatavari), one of the well-known medicinal plants, is known to prevent aging, increase longevity and impart immunity. ${ }^{11}$ Isoprinosine is a synthetic purine derivative with immunomodulatory and antiviral properties. It is an antiviral drug used as an immunomodulator, treatment for cancer, age-associated immune impairments and AIDS. ${ }^{12}$

Our study aimed to evaluate the radio-mitigating property of Asparagus racemosus root ethanolic extract (ARE) and Isoprinosine (IPR) in swiss albino mice.

\section{MATERIALS AND METHODS}

\section{Collection of plant material}

A. racemosus roots were collected from Coorg district and were identified by a Taxonomist from Mangalore University, Karnataka.

\section{Preparation of Root Extract}

Asparagus racemosus (AR) roots were washed in distilled water, air dried and then dried in hot air oven at $40^{\circ}-50^{\circ} \mathrm{C}$ for a week. The dried plant material was powdered using mixer grinder and subjected to Soxhlet extraction with $99 \%$ ethanol for $48 \mathrm{hr}$. The mixture was evaporated to dryness in a rotary flash evaporator (Superfit rotavap, Model: PBU - 6) and stored in a refrigerator until further use.

\section{Procurement of Isoprinosine}

Isoprinosine tablets (product code: 78/158) were procured from Brandmedicines, European Union.

\section{Ethical clearance}

The Institutional Animal Ethics Committee of K S Hegde Medical Academy, NITTE (Deemed to be University) approved this study (Ref. KSHEMA/IAEC/19/2015 dated 27.11.2015).

The entire study was carried out at the Central Research Laboratory, K.S Hegde Medical Academy, NITTE (Deemed to be University), Mangaluru, Karnataka.

\section{Post-radiation study}

Radiation exposure was carried out at the Microtron center, Mangalore University using the linear accelerator. The animals were restrained in well-ventilated perspex box and exposed to sublethal dose (6Gy) of whole - body electron beam radiation (EBR) at a distance of $100 \mathrm{~cm}$ from the beam exit point of the linear accelerator and at a dose rate of $3 \mathrm{~Gy} /$ min.

The experimental animals were divided into four groups of six animals, each as follows:

Group I - Control: administered orally with vehicle for 15 consecutive days
Group II - Post-radiation control (Post RC): administered orally with vehicle for 15 consecutive days after exposure to sublethal dose (6 Gy) EBR.

Group III - ARE $200 \mathrm{mg} / \mathrm{kg}$ b.wt: administered orally with asparagus root extract for 15 consecutive days after irradiation.

Group IV - IPR - $400 \mathrm{mg} / \mathrm{kg}$ b.wt: administered orally with Isoprinosine for 15 consecutive days after irradiation.

The optimum drug dosage for ARE and IPR was selected based on our previous toxicity (acute and sub-acute) study which was carried out following OECD guidelines. From the results of this study it was found that the No Observed Adverse Effect Level (NOAEL) of ARE and IPR was greater than $2000 \mathrm{mg} / \mathrm{kg}$ body weight. ${ }^{13}$ Effect of ARE and IPR on 30 -day survival against lethal radiation dose (10Gy) was determined in our previous study, in which it was found that ARE - 200mg/kg b.wt and IPR - $400 \mathrm{mg} / \mathrm{kg}$ b.wt increased the 30-day survival of the irradiated mice and hence these doses were considered for further radiation experiments. ${ }^{14}$

The animals were housed in the animal house and exposed to a sublethal dose of radiation before the administration of ARE and IPR. Throughout the study period, food and water intake were recorded daily, whereas, body weight was recorded once in a week.

\section{Assessment of hematological parameters}

At the end of the experimental period, the animals were sacrificed by cervical dislocation; blood samples were collected in Ethylene Diamine Tetra acetic acid (EDTA) coated vials for the analysis of hematological parameters such as WBC count, RBC count, Hb levels, lymphocytes (LY\%), monocytes (MO\%), granulocytes (GR\%) and platelet (PLT) count using Veterinary Blood Cell Counter (ERMA INC. model: PCE210VET).

The results obtained in the treatment groups were compared with the control and radiation control groups.

\section{Biochemical analysis}

A part of the blood was collected in plain vials to separate serum. Blood was centrifuged at $3000 \mathrm{rpm}$ at room temperature to separate the serum. Serum was collected and used for the estimation of lipid peroxidation according to the method of Buege and Aust ${ }^{15}$ and Total antioxidant capacity by phospho-molybdenum method as described by Prieto et al. ${ }^{16}$ using Visible Spectrophotometer (Systronics 2200).

\section{Estimation of cytokines level}

Liver tissue was excised from the experimental animals.10\% of liver homogenate was prepared in phosphate buffer saline (PBS) using a glass homogenizer. It was centrifuged at $5000 \mathrm{rpm}$ for $15 \mathrm{~min}$ at $4^{\circ} \mathrm{C}$. The supernatant was used for the estimation of cytokines namely, Interleukin-2 (IL-2), Interferon- $\gamma$ (IFN- $\gamma$ ) and Tumor necrosis factor$\alpha$ (TNF- $\alpha$ ) using mouse Enzyme-linked immunosorbent Assay (ELISA) kits (Koma biotech inc.) following manufacturer's instructions in an ELISA reader (Make: Rapid Diagnostics).

\section{Statistical analysis}

The results obtained were expressed as Mean \pm SD. One-Way Analysis of variance (ANOVA) was used to make a statistical comparison of hematological and biochemical analysis between the Control, radiation control and treatment groups followed by Tukey's multiple comparison test using graph pad Prism 7.0 software. The criterion for statistical significance was set at $p<0.05$. 


\section{RESULTS}

\section{Effect of ARE and IPR post-treatment on hematological parameters}

The Post-radiation control group showed a statistically significant reduction in the hematological parameters - WBC count, Hb levels $(p$ $<0.05$ ) percentage of Monocytes, Granulocytes and Platelet Count $(p<$ 0.001 ) compared to control group.

IPR post - treatment group showed a significant increase in percentage of lymphocytes $(p<0.05)$ and monocytes $(p<0.001)$ compared to post radiation control. ARE and IPR post-treatment caused a statistically significant increase in platelet count $(p<0.001)$ compared to post radiation control (Table 1).

\section{Effect of ARE and IPR post-treatment on Lipid peroxidation and total antioxidant capacity}

Irradiation of mice resulted in a significant elevation in lipid peroxidation $(p<0.01)$ compared to normal control. Post-treatment of mice with ARE and IPR after radiation exposure caused significant depletion in lipid peroxidation $(p<0.05)$ (Figure 1). Irradiation of mice caused a significant decrease $(p<0.001)$ in the level of serum total antioxidant capacity (TAC) level compared to the normal control mice. Administration of ARE to the animals after irradiation caused a significant elevation in

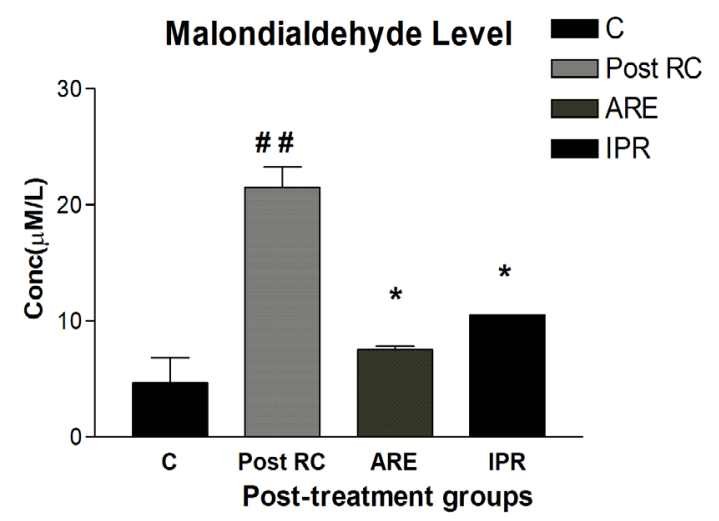

Figure 1: Chemical structure of a) Conessine and b) Aripiprazole. (C - Control, Post-RC - Post-radiation Control, ARE-Asparagus root ethanolic extract, IPR Isoprinosine. Values are expressed as Mean \pm SD $(n=24),{ }^{*} p<0.05$ ARE and IPR vs Post $-\mathrm{RC}$, \# $p<0.01$ Post-RC vs $\mathrm{C}$ )

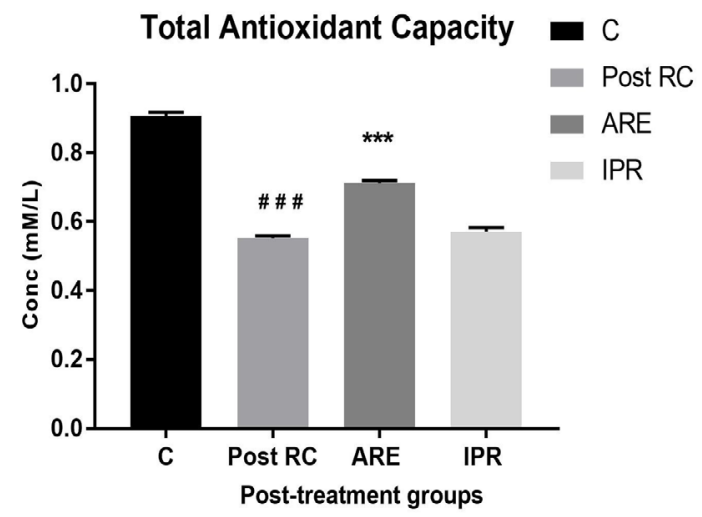

Figure 2: TAC level in post-treatment groups.

(C - Control, Post-RC - Post-radiation Control, ARE-Asparagus root ethanolic extract, IPR- Isoprinosine, Values are expressed as Mean \pm SD $(n=24),{ }^{*} p<0.05$ ARE and IPR vs Post RC, \#\#p<0.01 ( vs Post-RC)
TAC level $(p<0.001)$ when compared to the irradiated group. IPR posttreatment did not show any substantial change in the decreased level of TAC (Figure 2).

\section{Effect of ARE and IPR post-treatment on cytokines level}

The cytokines level in the liver homogenate of irradiated mice showed that IL-2, TNF- $\alpha$ and IFN- $\gamma$ significantly increased $(p<0.001, p<0.05$ and $p<0.001)$ after exposure to whole-body sublethal dose (6 Gy) of radiation when compared with the normal control group. Administration of ARE and IPR after irradiation significantly reduced $(p<0.01)$ the levels of IL- 2 and TNF- $\alpha$ in the post-treated animals compared to postradiation control. An elevated level of IFN- $\gamma$ was significantly reduced ( $p$ $<0.05)$ in the IPR post-treatment animals whereas ARE post-treatment did not cause any significant reduction in the IFN- $\gamma$ level compared to post-radiation control (Figure 3-5).

\section{DISCUSSION}

The agents delivered during or shortly after radiation exposure to repopulate a critical cell compartment are termed as the radiation mitigators which are used to prevent acute toxicity. Much of the focus of mitigators has been in the field of developing chemo-preventatives to reduce carcinogenesis of total body exposures during radiologic terrorism and space research.

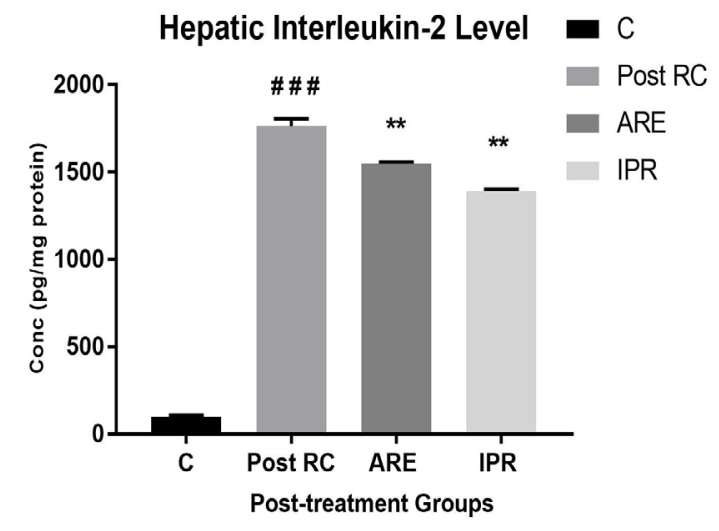

Figure 3: IL-2 level in post-treatment groups.

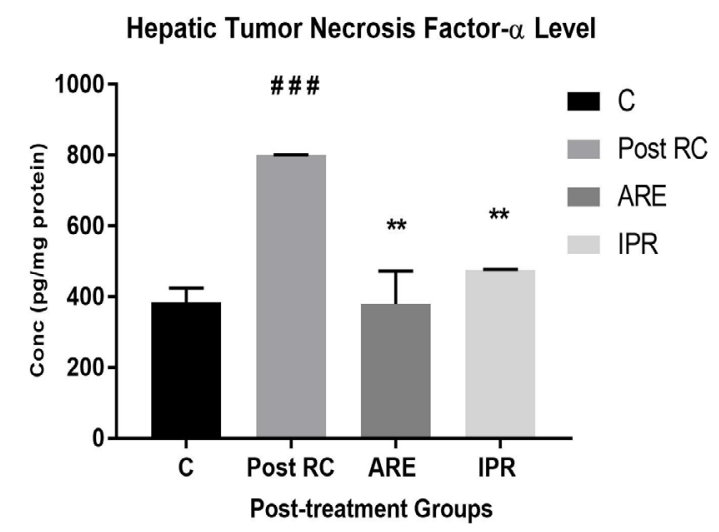

Figure 4: TNF-a level in post-treatment groups. 


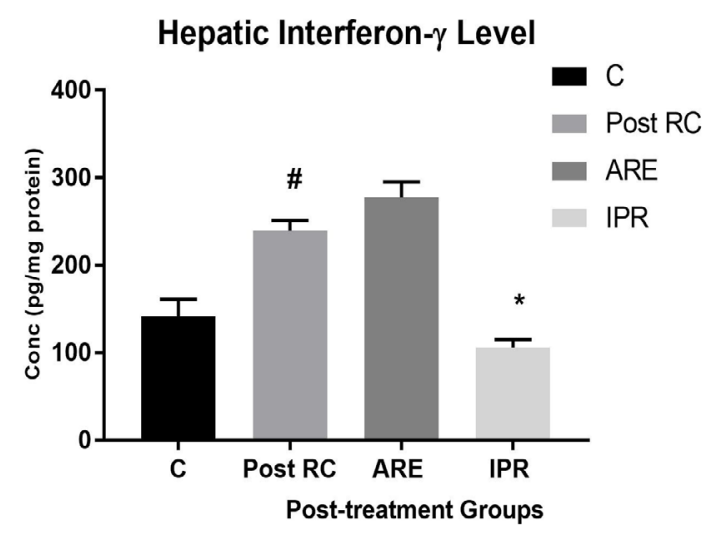

Figure 5: IFN $-\gamma$ level in post-treatment groups.

(Figure 3-5: C-control, Post RC- Post-radiation control, ARE - Asparagus root ethanolic extract, IPR - Isoprinosine. Values are expressed as Mean \pm SD $(n=24)$, ${ }^{*} p<0.05$ IPR vs Post RC group; \# $p<0.05$, \#\#\# $p<0.001$ Post RC vs Control).

Plant-derived antioxidant compounds have received considerable attention because of their physiological effect like antioxidant, antiinflammatory, antitumor activities and low toxicity compared with those of synthetic phenolics. ${ }^{17,18}$ Through our systematically comparative study, the ethanolic Asparagus racemosus root extract was found to be an excellent free-radical scavenger and a potent natural antioxidant. ${ }^{19}$

The hematopoietic system is among the most radiosensitive in the body as it has the highest cell turnover. In the present study, the decline in hematological constituents may be attributed to direct destruction of mature circulating cells, loss of cells from the circulation by hemorrhage, or leakage through capillary walls and loss of production of cells by radiation. ${ }^{20}$ The recovery of hematological constituents by ARE and IPR post-administration may be attributed to the stimulation of the proliferation of hematopoietic stem cells and also significant protection of erythropoietic cells in bone marrow.

Elevation in lipid peroxidation takes place as a result of histopathological changes in the liver including dilatation of blood vessels, congestion in the lobules, enlargement of portal areas and infiltration of mixed inflammatory cells around the necrotic hepatocytes and the portal area. These results were found to be in agreement with the study findings of Burlakova (2004) who reported that exposure to $\gamma$-irradiationinduced liver lesion was associated with a massive elevation in liver Malondialdehyde (MDA) level. ${ }^{21}$ From the previous study of Tyurina et al. (2008) the increase in MDA could be due to the lipid peroxidation induced by ionizing radiation through the radiolysis of water in the aqueous environment of the cells leading to the production of hydroxyl radicals $(\bullet \mathrm{OH})$. When these hydroxyl free radicals interact with the polyunsaturated fatty acids in the lipid portion of biological membranes, they initiate the lipid peroxidation and finally damage the cell membranes. ${ }^{22}$

The majority of the studies have revealed that exposure to wholebody ionizing radiation decreased the total antioxidant capacity of an organism and depleted known antioxidants such as GSH, ascorbic acid and urea. ${ }^{23}$ The free radicals generated by the radiolysis of water within the cell is responsible for these harmful effects. ARE and IPR post-treatment reduced the MDA level indicating a decrease in lipid peroxidation. Also, ARE post-administration was efficient in increasing the total antioxidant capacity compared to radiation control group whereas, in the IPR post - treatment group no significant increase was observed in the total antioxidant capacity compared to radiation control group. The observed effects may be partly attributed to the scavenging of radiation-induced free radicals as well as by increasing the endogenous antioxidant levels. Inhibition of radiation-induced apoptosis, which is mediated by membrane lipid peroxidation, may play a role in radio mitigating activity of ARE and IPR.

According to literature, determination of protein cytokines in tissues reflects the actual function of cytokines. Such method provides an important tool for studying the local protein cytokines in sickness behavior and immune-mediated diseases as well as to determine local therapeutic efficacy of immunomodulatory drugs. ${ }^{24}$

In the post-radiation study, it was found that IL - 2, TNF- $\alpha$ and IFN - $\gamma$ increased after exposure to whole-body sublethal dose (6 Gy) radiation. Administration of ARE and IPR after irradiation significantly reduced the levels of IL - 2 and TNF - a compared to post - radiation control. Elevated level of IFN - $\gamma$ was reduced considerably in the IPR treated animals, whereas ARE treatment after radiation exposure did not cause any significant reduction in the IFN $-\gamma$ level compared to radiation control. The observed results may be due to oxidative stress and free radicals formed due to radiation exposure that triggers non - specific, acute reaction termed as 'cytokine storming. Release of these cytokines and other protein products of activated transcription factors may play a major role in subsequent radiation-induced inflammation and tissue damage. ${ }^{25}$

One of the mechanisms for the increase in pro-inflammatory cytokines, which is caused due to inflammation following radiation exposure, may be attributed to High Mobility Group Box I (HMGB1) pathway. It is well established that, as the oxidative stress increases, accumulation of unrepaired intracellular damage is observed which causes the cells

Table 1: Effect of ARE and IPR post-treatment on hematological parameters in swiss albino mice.

\begin{tabular}{ccccc}
\hline Parameter & \multicolumn{3}{c}{ Groups } \\
\hline Control & Post-RC & ARE & IPR \\
\hline RBC & $10.55 \pm 0.65$ & $8.88 \pm 1.79$ & $10.41 \pm 1.53$ & $9.21 \pm 2.75$ \\
WBC & $10.4 \pm 0.42$ & $5.23 \pm 1.58^{*}$ & $5.7 \pm 1.47$ & $7.57 \pm 1.12$ \\
Hb & $14.8 \pm 0.45$ & $11.96 \pm 2.62^{*}$ & $14.46 \pm 2.05$ & $12.56 \pm 3.99$ \\
LY \% & $63.76 \pm 11.62$ & $85.9 \pm 8.17$ & $81.16 \pm 8.75$ & $58.23 \pm 8.22^{*}$ \\
GR \% & $24.36 \pm 2.92$ & $5.83 \pm 1.07^{* \# *}$ & $8.3 \pm 1.30$ & $16.66 \pm 1.10$ \\
MO \% & $14.96 \pm 4.73$ & $5.0 \pm 1.30^{*}$ & $12.6 \pm 0.70$ & $26.9 \pm 1.41^{* * *}$ \\
PLT & $516 \pm 76.17$ & $170.66 \pm 50.57^{\# * *}$ & $556.5 \pm 16.26^{* * *}$ & $467 \pm 44.22^{* * *}$ \\
\hline
\end{tabular}

Values are expressed as Mean \pm SD $(n=24) .{ }^{*} p<0.05,{ }^{* *} p<0.001$ ARE and IPR vs Post-RC, (C - Control, Post-RC - Post-radiation Control, ARE-Asparagus root ethanolic extract, IPR- Isoprinosine; \# $p<0.05$,\#\#\# $p$ $<0.001$ Post - RC vs Control). 
Poonacha, et al.: Radio-mitigating Property of Asparagus racemosus Root Extract and Isoprinosine in Swiss Albino Mice

to undergo either programmed cell death (i.e. apoptosis) or necrosis (a complete disintegration of cellular components which causes complete cell lysis). Apoptosis is an energy-consuming process involving the suicidal breakdown of a group of cells. The damaged apoptotic cells do not cause inflammation as they are engulfed/phagocytosed by macrophages. In contrast, severely damaged cells that are unable to form phagosome mainly due to lack of energy (ATP), undergo necrosis and disintegrate the intracellular components, which augments inflammation. Several factors, such as the ratio of ROS level to antioxidant level, energy loss, failure in the repair process of damaged DNA lesions and irreversibly disrupted permeability of cellular membrane influences the susceptibility of the individual cell towards necrotic cell death. ${ }^{26}$

\section{CONCLUSION}

From our study, it is evident that ARE and IPR exhibit radiomitigating effects that occur through several mechanisms, such as free radical scavenging, anti-inflammation, facilitation of repair activity, regeneration of hematopoietic cells and affecting to molecular levels. These mechanisms are involved in radiation response and tissue damage repair, which may be potential pharmacological targets for the development of ideal radio-mitigators.

\section{ACKNOWLEDGEMENT}

The authors would like to extend heartfelt gratitude to the Board of Research in Nuclear Sciences, Department of Atomic Energy, Government of India for providing the financial support (Sanction No. 34(1)/14/32/2014-BRNS). The authors remain grateful to NITTE (Deemed to be University) for providing laboratory facilities and also would like to thank Dr.K.R Chandrashekar, Chairman, Department of Applied Botany, Mangalore University, Karnataka, India for the identification of the study plant.

\section{CONFLICT OF INTEREST}

The authors declare no Conflict of interest.

\section{ABBREVIATIONS}

-OH: Hydroxyl radical; AIDS: Acquired Immuno Deficiency Syndrome; ANOVA - One: Way Analysis of Variance; ARE: Asparagus racemosus ethanolic root extract; ATP: Adenosine Triphosphate; C: Control; DNA: Deoxyribonucleic Acid; EBR: Electron Beam Radiation; EDTA: Ethylene Diamine Tetra - acetic Acid; ELISA: Enzyme Linked Immunosorbent Assay; GR \%: Granulocyte Percentage; GSH: Reduced Glutathione; Gy: Gray; Hb: Hemoglobin; HMGB 1: High Mobility Group Box 1; IFN $\gamma$ : Interferon Gamma; IL - 2: Interleukin - 2; IPR: Isoprinosine; Ly\%: Lymphocyte Percentage; MDA: Malondialdehyde; MO\%: Monocyte Percentage; NOAEL: No Observed Adverse Effect Level; OECD: Organization for Economic Co-operation and Development; PBS: Phosphate Buffered Saline; PLT: Platelet; RBC: Red Blood Corpuscle; RC: Radiation Control; SD: Standard Deviation; TAC: Total Antioxidant Capacity; TNF - $\mathbf{\alpha}$ : Tumor Necrosis Factor alpha; WBC: White Blood Corpuscle.

\section{REFERENCES}

1. Saha GB. Radiation biology in physics and radiobiology of nuclear medicine. New York: Springer Science + Business Media. 2013
2. Neill OP, Fielden EM. Primary radical process in DNA. Adv Radiat Biol. 1993; 17:53-120.

3. Dillman RO. Radioimmunotherapy of B-cell lymphoma with radiolabelled antiCD20 monoclonal antibodies. Clin Exp Med. 2006;6(1):1-12.

4. Greenberger JS, Anderson J, Berry LA, Epperly M, Cronkite EP, Boggs SS. Effects of irradiation of CBA/CA mice on hematopoietic stem cells and stromal cells in long-term bone marrow cultures. Leukemia. 1996;10(3):514-27.

5. Hosseinimehr SJ. Trends in the development of radioprotective agents. Drug Dis Today. 2007;12(19-20):794-805.

6. Arora R, Gupta D, Chawla R, Sagar R, Sharma A, Kumar R, et al. Radioprotection by plant products: Present status and future prospects. Phytother Res. 2005;19(1):1-22

7. Ross GM. Induction of cell death by radiotherapy. Endocr Relat Cancer .1999;6(1):41-4

8. Citrin D, Cotrim AP, Hyodo F, Baum BJ, Krishna MC, Mitchell JB. Radioprotectors and Mitigators of Radiation- Induced Normal Tissue Injury. Oncologist. 2010;15(4):360-71.

9. Jagetia GC. Radioprotective Effects of Plants and Herbs against the Effects of Ionizing Radiation. J Clin Biochem Nutr. 2007;40(2):74-81.

10. Finch PW, Rubin JS. Keratinocyte growth factor/fibroblast growth factor 7 , a homeostatic factor with therapeutic potential for epithelial protection and repair. Adv Cancer Res. 2004;91:69-136.

11. Visavadiya NP, Soni B, Soni B, Madamwar D. Suppression of reactive oxygen species and nitric oxide by Asparagus racemosus root extract using in vitro studies. Cell and Mol Biol. 2009:55:1083-95.

12. Huttenlocher PR, Mattson RH. Isoprinosine in subacute sclerosing panencephalitis. Neurology. 1979;29(6):763-71.

13. Bhandary BSK, Sharmila KP, Kumari NS, Bhat VS, Fernandes R. Acute and Subacute Toxicity Profile of Asparagus racemosus root extract, Isoprinosine and Shatvari Syrup in Swiss Albino Mice. J App Pharm Sci. 2017;7(05):129-35.

14. Sharmila KP, Bhandary SKB, Fernandes R, Kumari NS, Bhat VS, Shetty JK, et al. Radioprotective Potential of Asparagus racemosus root extract and Isoprinosine against Electron beam radiation induced Immunosupression and Oxidative stress in Swiss Albino mic. J Nat Sc Biol Med. 2018;9(2):242-6.

15. Buege JA, Aust SD. Microsomal Lipid Peroxidation. Methods Enzymol. 1978;52:302-10

16. Prieto P, Pineda M, Aguilar M. Spectrophotometric quantitation of antioxidant capacity through the formation of a phosphomolybdenum Complex: Specific application to the determination of vitamin E. Anal Biochem. 1999;269(2):33741.

17. Aruna P, Debra L, Rateri, Shubin KS, Sibu, Alan D. Citrullus lanatus - sentinel (watermelon) extract reduces atherosclerosis in LDL receptor-deficient mice. J Nutr Biochem. 2012;107-21.

18. Kokate CK, Practical Pharmacognosy, $4^{\text {th }}$ ed. New Delhi: Vallabh Prakashan. 2008;107-11.

19. Bhandary SK, Sharmila KP, Suchetha KN, Vadisha SB. Phytochemical Profile and In vitro Antioxidant activity of Asparagus racemosus Root Extract, Isoprinosine and Shatavari Syrup. JOHR. 2015;3(4):215-23.

20. Daga SS, Jain VK, Goyal PK. Radio response of leucocytes in peripheral blood of mice against gamma-radiation and their protection by Liv 52. Probe. 1995;34:222-6.

21. Burlakova EB, Naidikh VI. The Effects of Low Dose Radiation. Boston: VSP Utrecht. 2004.

22. Tyurina YY, Tyurin VA, Epperly MW, Greenberger JS, Kagan VE. Oxidative lipidomics of $\gamma$-irradiation-induced intestinal injury. Free Rad Biol Med. 2008;44(3):299-314.

23. Karbownik M, Reiter RJ. Antioxidative effects of melatonin in protection against cellular damage caused by ionizing radiation. Proceedings Society Exp Bio Med. 2000;225(1):9-22

24. Khalid ZM, Maha FT, Mona AB, Yousef AB. Measurement of protein cytokines in tissue extracts by enzyme-linked immunosorbent assays: Application to lipopolysaccharide-induced differential milieu of cytokines. Neuroendocrinol Let. 2005;26(3):231-6

25. Talas M, Szolgay E, Varteresz V. Influence of acute and fractional X-irradiation on induction of interferon in vivo. Arch Gesamte Virusforsch. 1972;38(2-3):143-8.

26. Samali A, Nordgren H, Zhivotovsky B, Peterson E, Orrenius S. A comparative study of apoptosis and necrosis in Hep G2 cells: Oxidant induced caspase inactivation leads to necrosis. Biochem Biophysic Res Commun. 1999;255(1):611.

Article History: Submission Date : 25-05-2020; Revised Date : 16-07-2020; Acceptance Date : 16-08-2020

Cite this article: Poonacha SK, Bavabeedu SKB, Nalilu SK, Pooja S, Bhat VS, Sanjeev G, et al. Asparagus racemosus Root Extract and Isoprinosine Exhibits Radio-mitigating Activity against lonizing Radiation-induced Detrimental Effects in Swiss Albino Mice. J Young Pharm. 2020;12(3):226-30. 